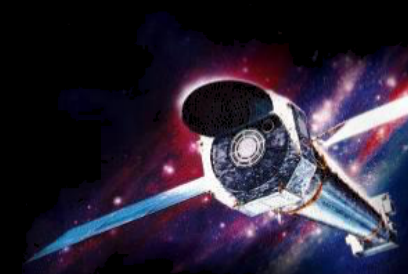

\title{
Modeling contamination migration on the Chandra X-ray Observatory - IV
}

Steve O'Dell, Doug Swartz, Neil Tice, Paul Plucinsky, Herman Marshall, Akos Bogdan, Catherine Grant, Allyn Tennant, Matt Dahmer

\author{
NASA Marshall Space Flight Center \\ Universities Space Research Association \\ Massachusetts Institute of Technology \\ Smithsonian Astrophysical Observatory \\ Northrop Grumman
}




\section{Outline}

$>$ Introduction

$>$ Molecular contamination on ACIS filters

$>$ Thermal model for ACIS cavity

$>$ Molecular transport simulations

$>$ Summary 


\section{Contamination-migration simulations for Chandra}

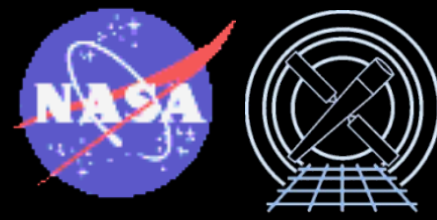

$>2005$ (I)

$>$ Geometric model (exterior)

口 Low-resolution geometric model for ACIS cavity

$>2013$ (II)

口 High-resolution geometric model for ACIS cavity

- Higher emissivity for contaminated surfaces

$>2015$ (III)

口 Same model as 2013

$>2017$ (IV)

- Extend geometric model into optical bench

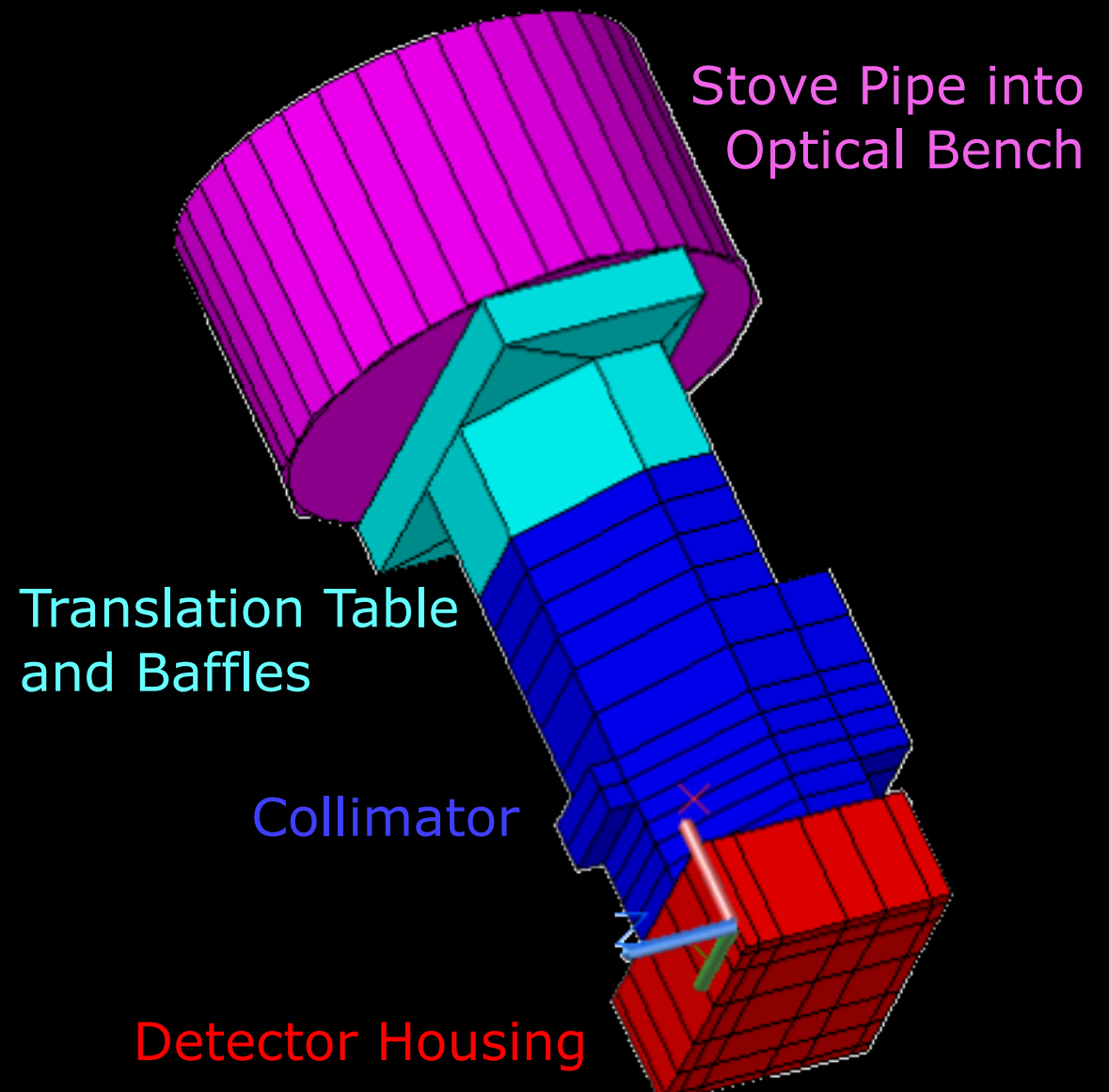




\section{Outline}

$>$ Introduction

$>$ Molecular contamination on ACIS filters

$>$ Thermal model for ACIS cavity

$>$ Molecular transport simulations

$>$ Summary 


\section{Thermal history}

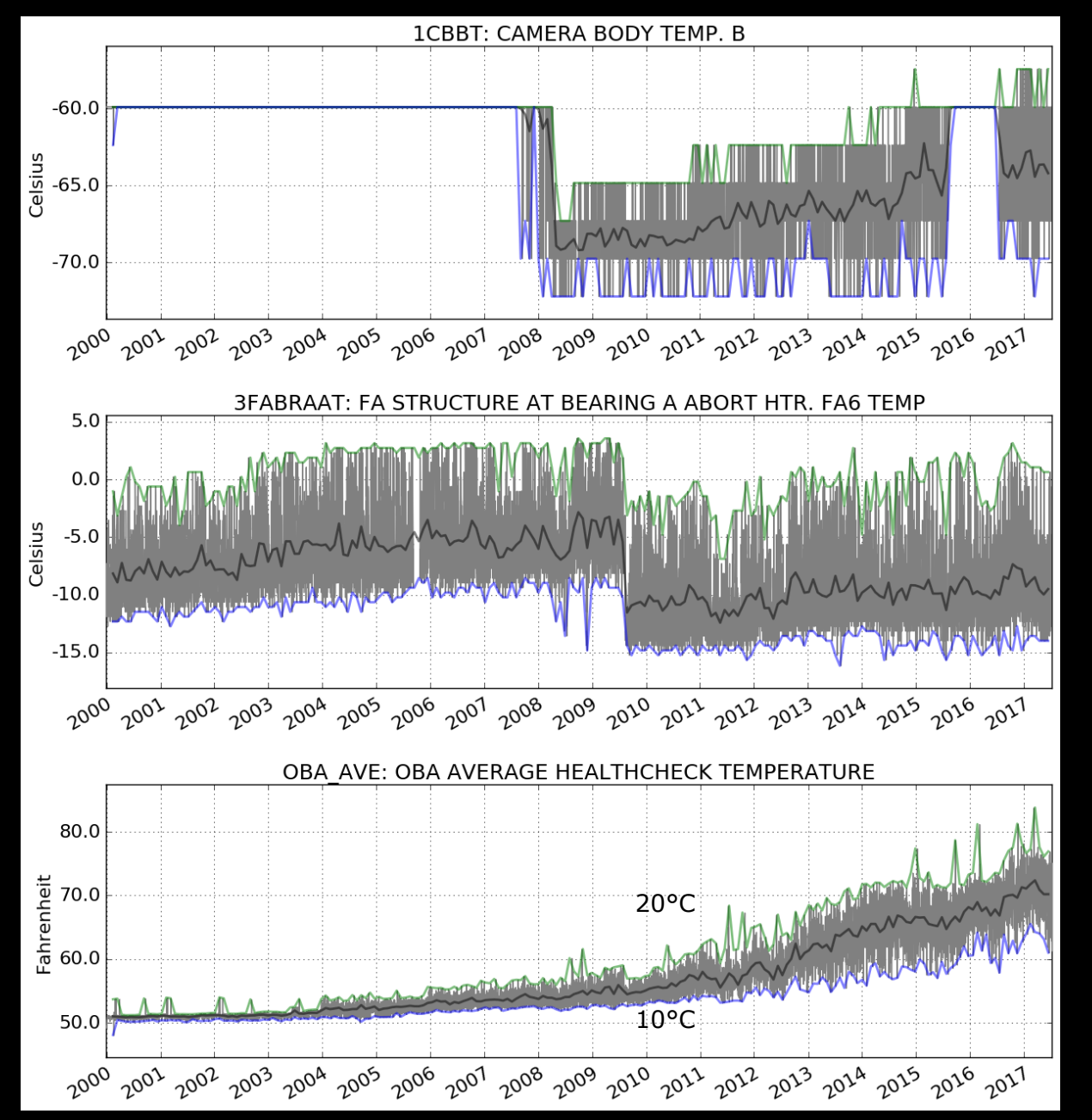

$>$ Most systems are warming

$\square$ Continuing degradation of external insulation (MLI)

$>$ Strive to keep ACIS focal plane cold to preserve performance $\square$ Carefully plan observations

口Disabled some heaters

- ACIS detector-housing heater (2008 April)

- A SIM focus-assembly heater (2009 August)

$>$ Optical Bench has warmed rapidly since about 2010

口 New contamination source? 


\section{Outline}

$>$ Introduction

$>$ Molecular contamination on ACIS filters

$>$ Thermal model for ACIS cavity

$>$ Molecular transport simulations

$>$ Summary 


\section{ACIS temperature distribution (exterior)}

$>\mathrm{T}_{\mathrm{DH}}=+25^{\circ} \mathrm{C}, \mathrm{T}_{\mathrm{FP}}=-60^{\circ} \mathrm{C}$

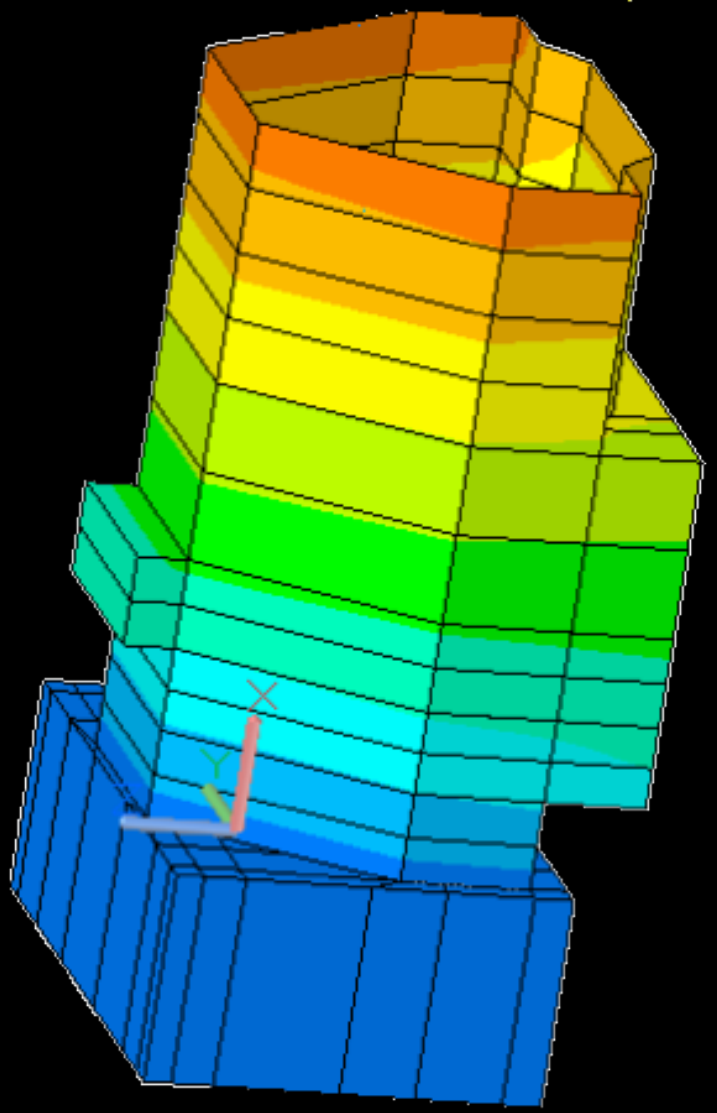

$$
\varepsilon_{\mathrm{OBF}}=0.40
$$

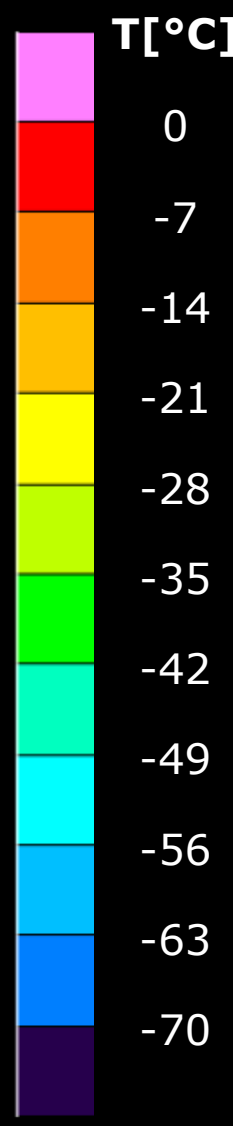

Modeling contamination migration on the Chandra X-ray Observatory - IV UV, X-Ray, and Gamma-Ray Space Instrumentation for Astronomy XX 2017 August 6-8, San Diego, CA USA
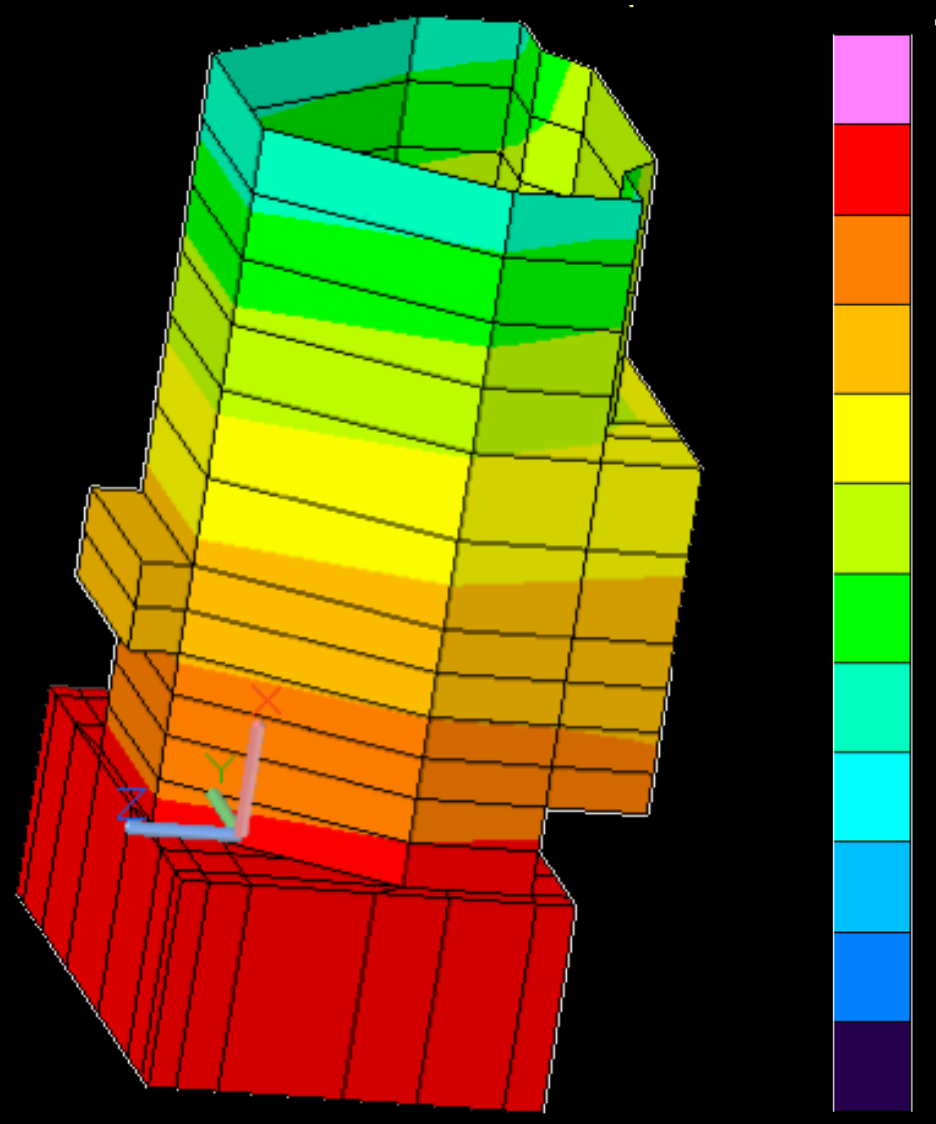

$\mathbf{T}\left[{ }^{\circ} \mathbf{C}\right]$

$+25$

$+20$

$+15$

$+10$

$+5$

0

$-5$

$-10$

$-15$

$-20$

$-25$
$\varepsilon_{\mathrm{OBF}}=0.40$
SPIE Conference 10397

Paper 13 Slide 9 


\section{ACIS temperature distribution (interior)}
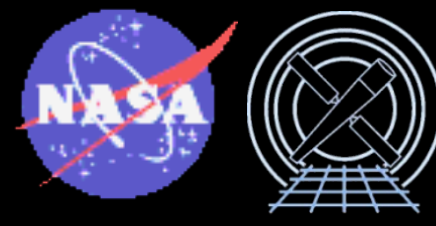

$>$ DH heater OFF, $T_{F P}=-120^{\circ} \mathrm{C}$

$>\mathrm{T}_{\mathrm{DH}}=+25^{\circ} \mathrm{C}, \mathrm{T}_{\mathrm{FP}}=-60^{\circ} \mathrm{C}$

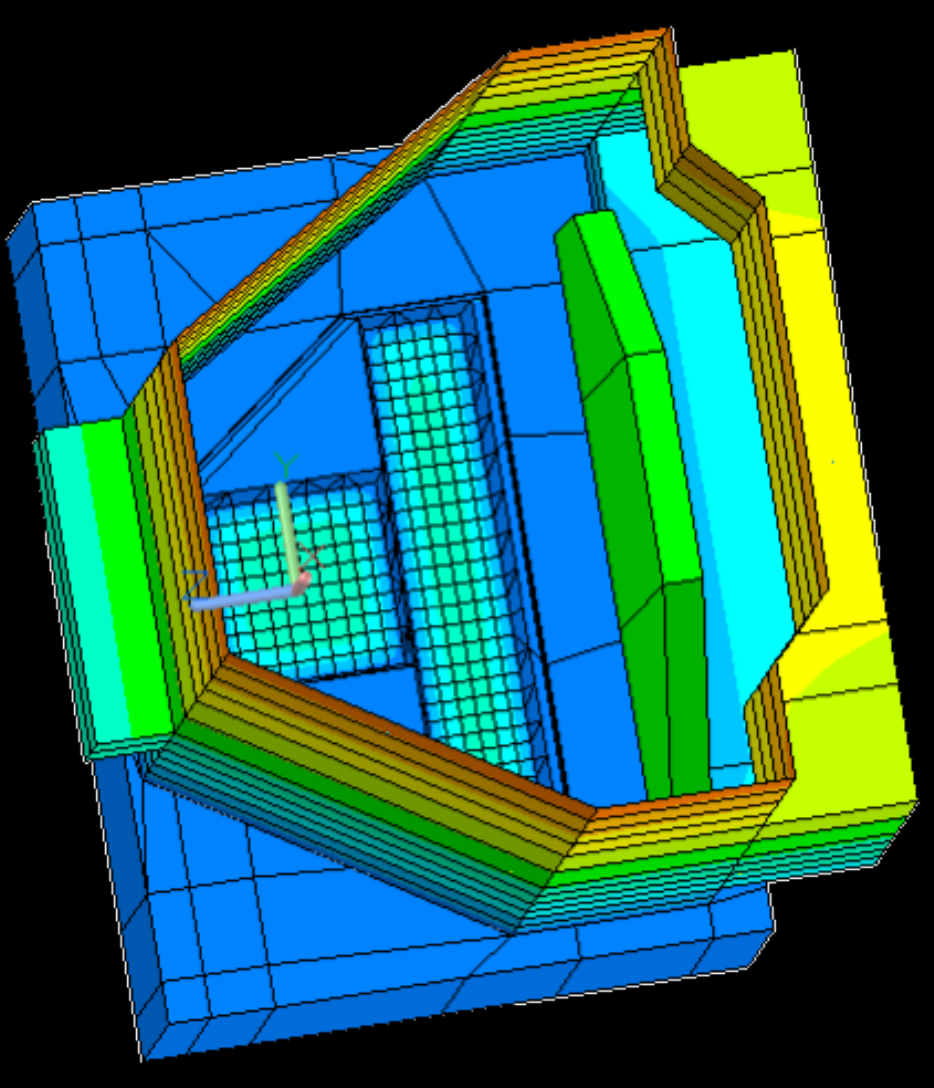

$$
\varepsilon_{\mathrm{OBF}}=0.40
$$
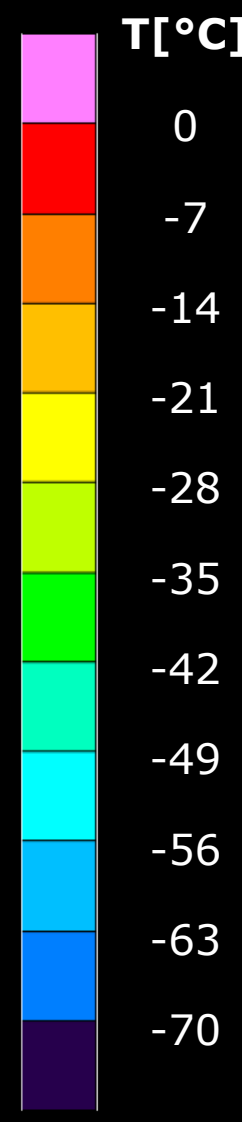

$\left[{ }^{\circ} \mathrm{C}\right]$
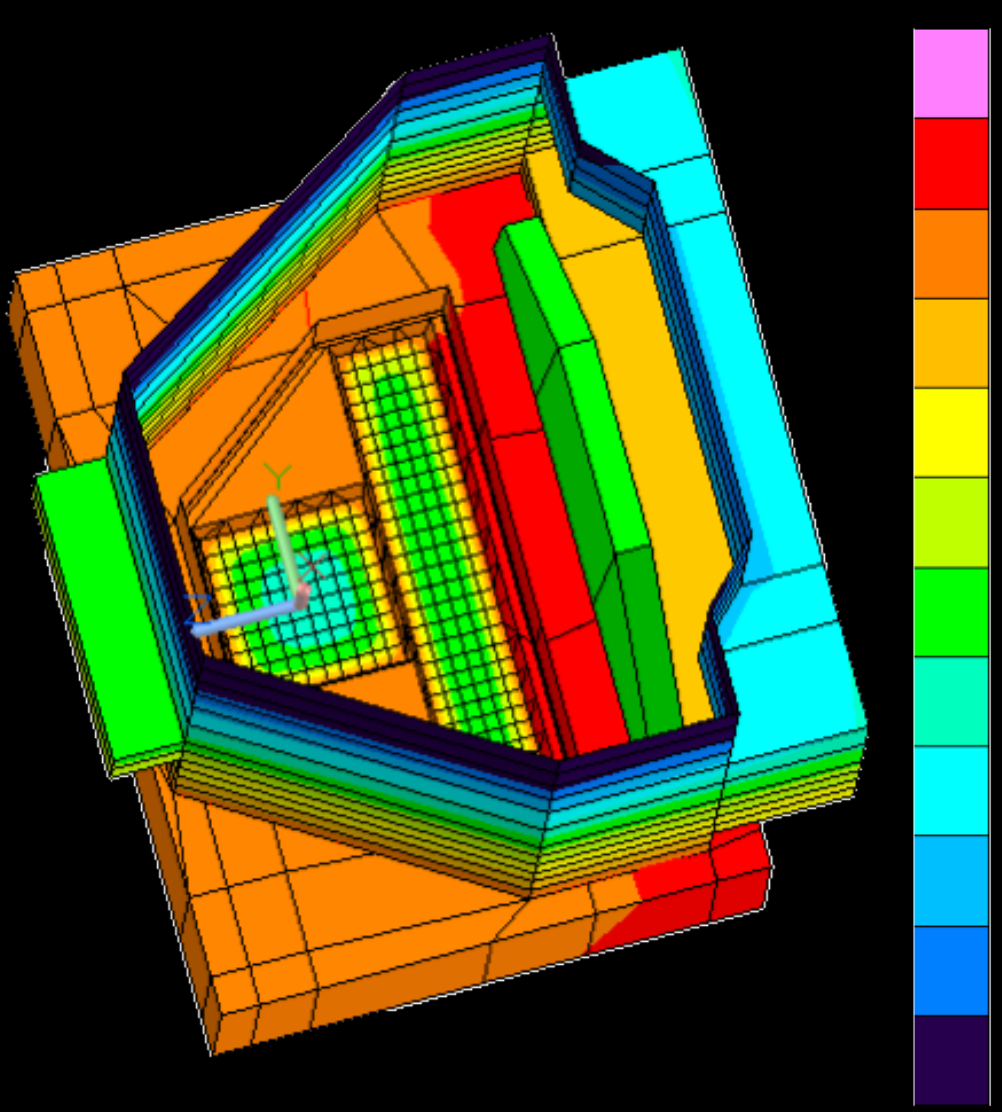

$$
\varepsilon_{\mathrm{OBF}}=0.40
$$

$\mathbf{T}\left[{ }^{\circ} \mathrm{C}\right]$

$+25$

$+22.5$

$+20$

$+17.5$

$+15$

$+12.5$

$+10$

$+7.5$

$+5$

$+2.5$

0
Modeling contamination migration on the Chandra X-ray Observatory - IV UV, X-Ray, and Gamma-Ray Space Instrumentation for Astronomy XX 2017 August 6-8, San Diego, CA USA
SPIE Conference 10397

Paper 13 Slide 10 


\section{Outline}

$>$ Introduction

$>$ Molecular contamination on ACIS filters

$>$ Thermal model for ACIS cavity

$>$ Molecular transport simulations

$>$ Summary 


\section{Molecular flux equations and geometric view factors}

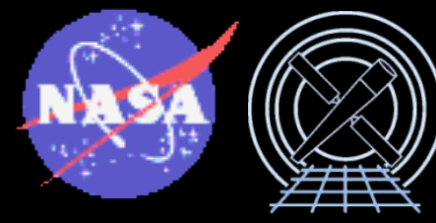

$>$ Net mass flux onto node $\mathrm{j}$

$$
\frac{d \mu_{j}}{d t}=-\dot{\mu}_{v}\left(T_{j}\right) \Theta\left(\mu_{j}\right)+\sum_{k} \dot{\mu}_{v}\left(T_{k}\right) \Theta\left(\mu_{k}\right) f_{j k} \frac{A_{k}}{A_{j}}
$$

$>$ Mass vaporization flux

口 Related to vapor pressure

$$
\dot{\mu}_{v}(T)=\frac{P_{v}(T)}{\sqrt{2 \pi R T / M}}
$$

$>$ Clausius-Clapeyron relation

- Temperature dependence

$\square$ Vaporization enthalpy $\Delta_{V} H$

$$
\begin{aligned}
& P_{v}(T)=P_{v}\left(T_{\circ}\right) \operatorname{Exp}\left[-\frac{\Delta_{v} H}{R}\left(\frac{1}{T}-\frac{1}{T_{\circ}}\right)\right] \\
& \dot{\mu}_{v}(T)=\dot{\mu}_{v}\left(T_{\circ}\right) \sqrt{\frac{T_{\circ}}{T}} \operatorname{Exp}\left[-\frac{\Delta_{v} H}{R}\left(\frac{1}{T}-\frac{1}{T_{\circ}}\right)\right]
\end{aligned}
$$

$>$ Geometric view factors

$$
f_{j k}=\boldsymbol{n}_{k} \cdot \Omega_{j k} / \pi
$$

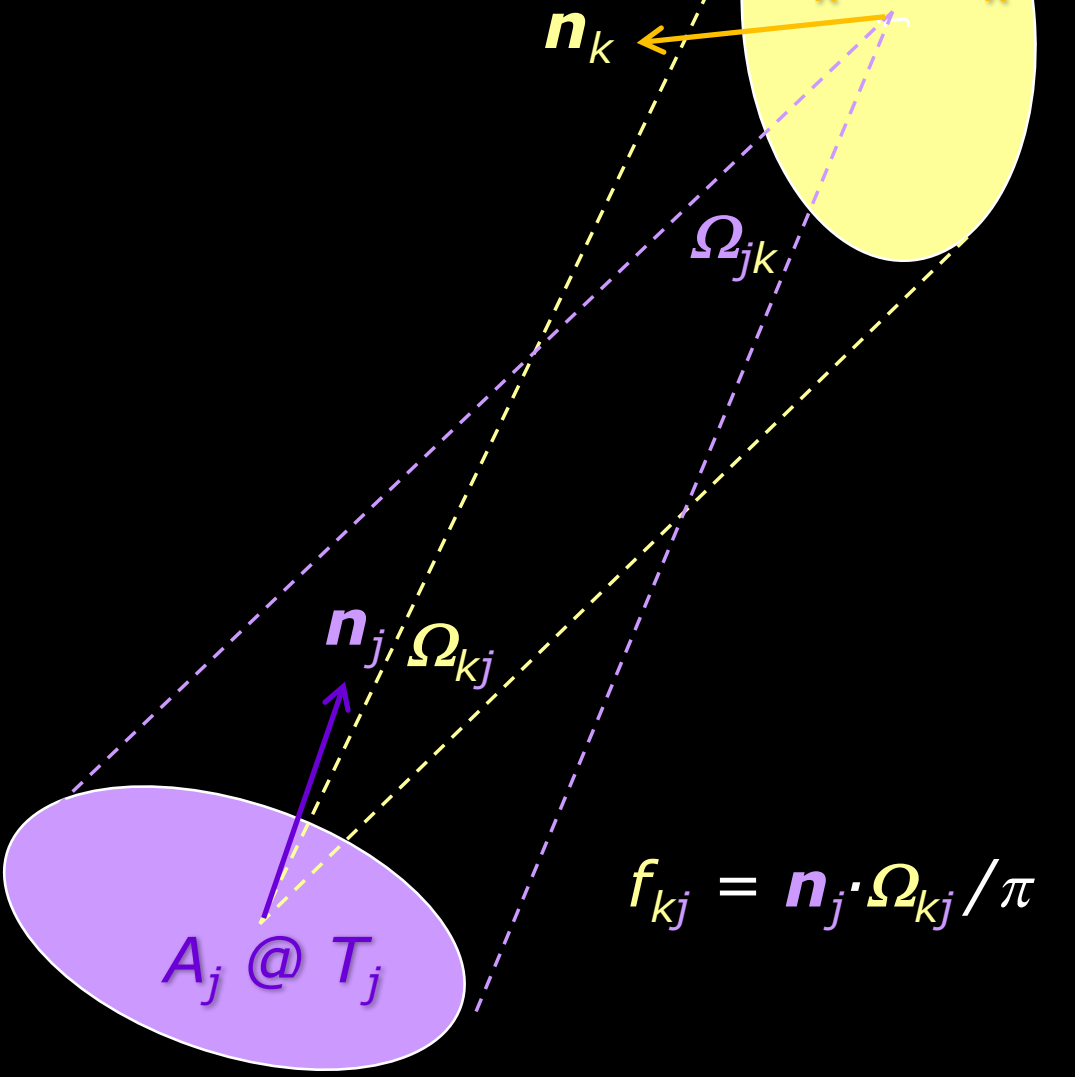

Modeling contamination migration on the Chandra X-ray Observatory - IV UV, X-Ray, and Gamma-Ray Space Instrumentation for Astronomy XX 2017 August 6-8, San Diego, CA USA

SPIE Conference 10397

Paper 13 Slide 12 


\section{Vaporization rate: Dependence upon phase state}

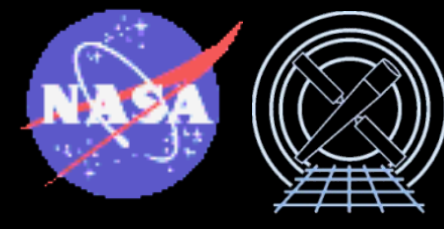

\section{Mass vaporization rates of a solid and of a liquid}

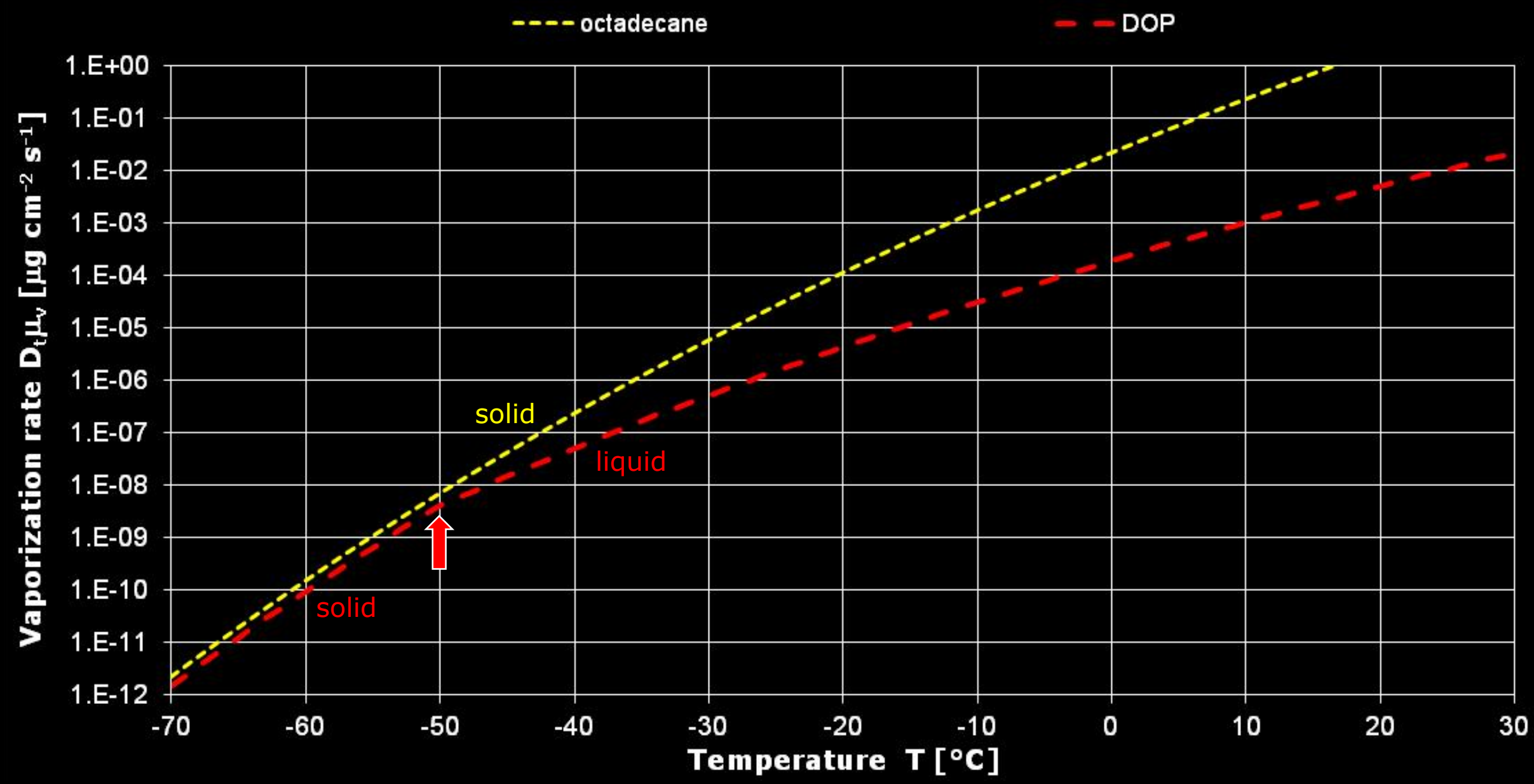

Modeling contamination migration on the Chandra X-ray Observatory - IV UV, X-Ray, and Gamma-Ray Space Instrumentation for Astronomy XX

SPIE Conference 10397 2017 August 6-8, San Diego, CA USA 


\section{Contaminant mass Cool bake-out}

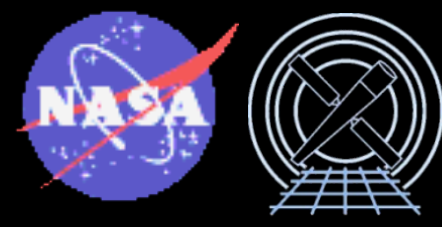

$$
\mathrm{T}_{\mathrm{FP}}=-60^{\circ} \mathrm{C} \quad \mathrm{T}_{\mathrm{DH}}=+25^{\circ} \mathrm{C} \quad \mathrm{T}_{\mathrm{OBF}}=+10^{\circ} \mathrm{C} \quad \mathrm{T}_{\pi}=-10^{\circ} \mathrm{C}
$$

$>$ Octadecane

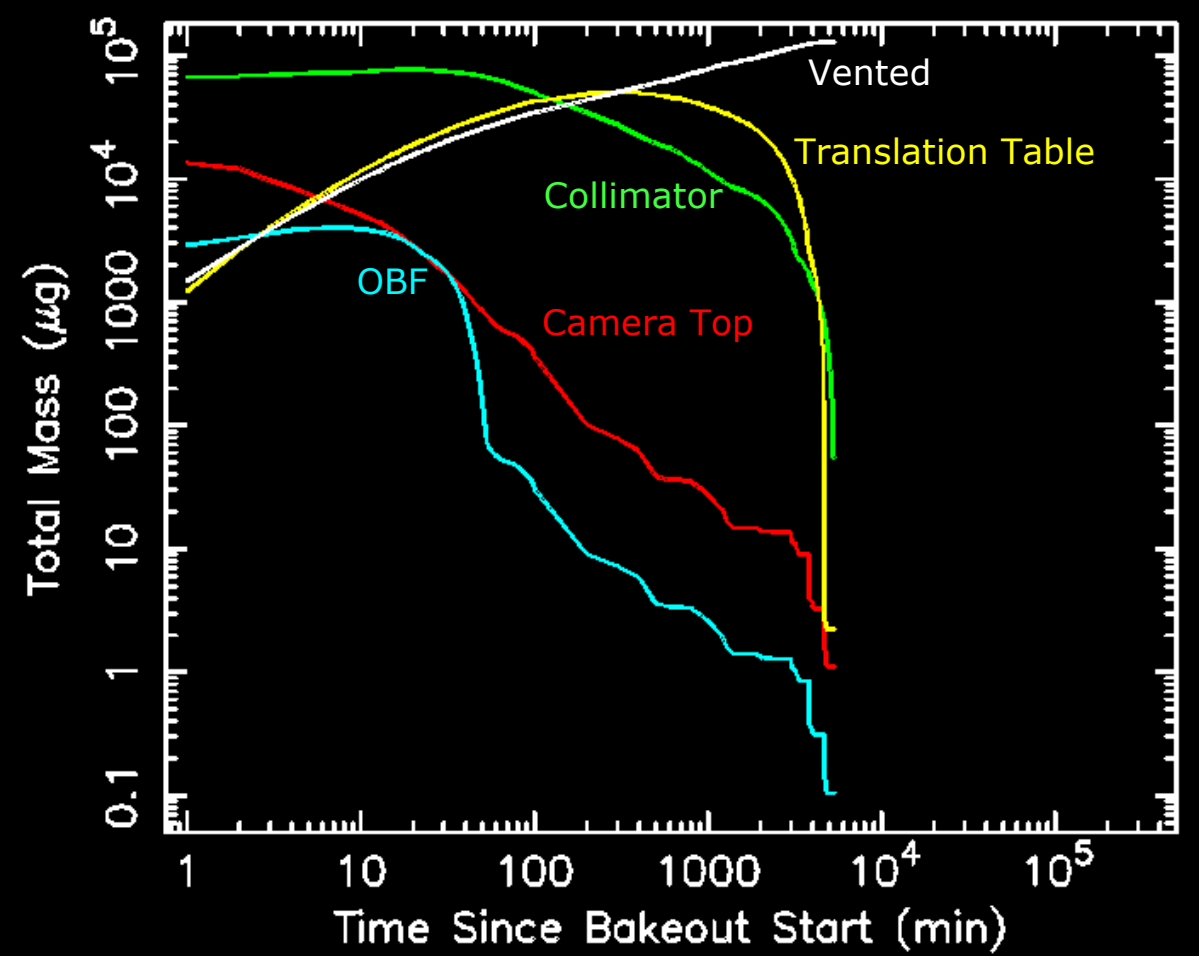

$>$ Dioctyl phthalate (DOP)

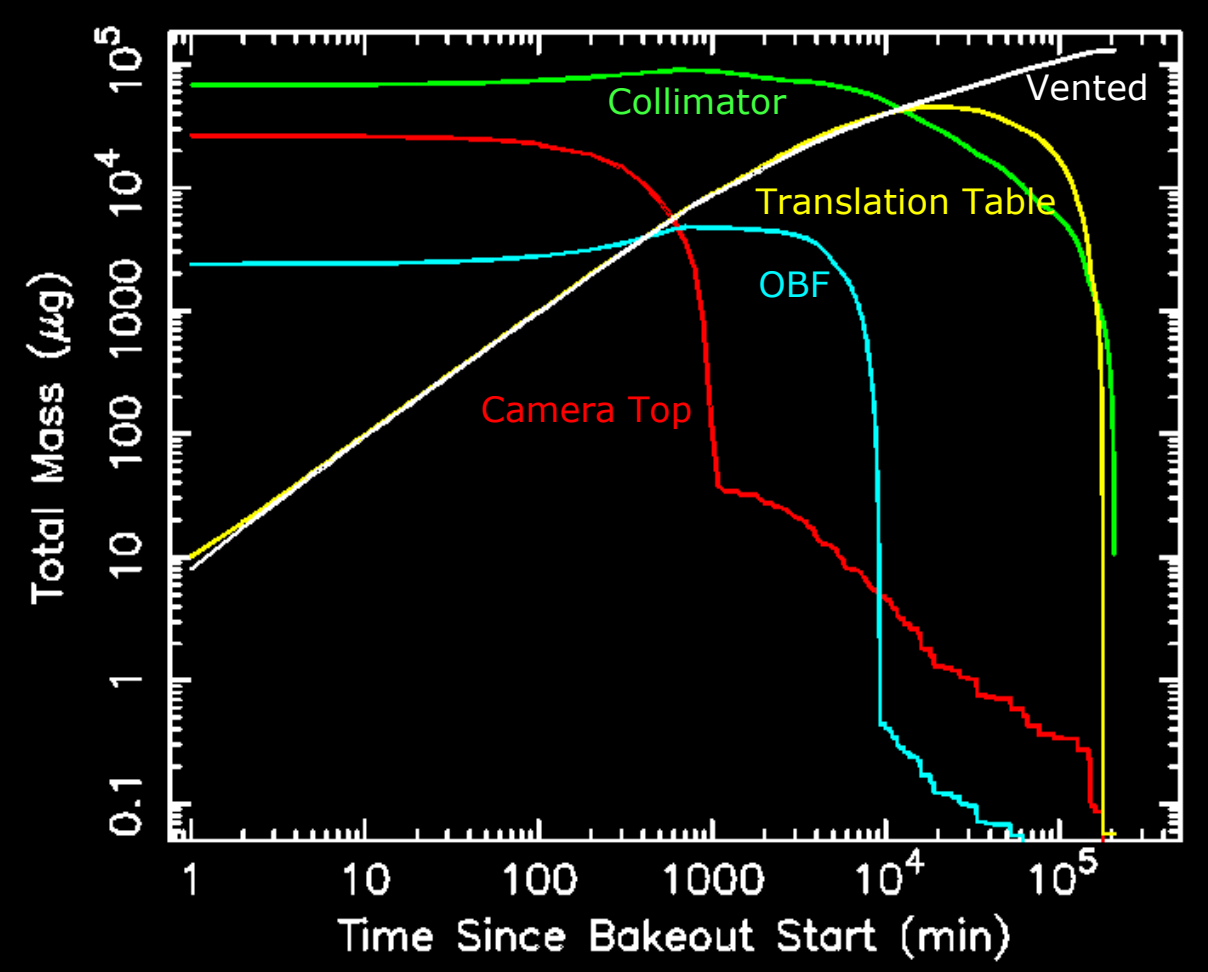

Modeling contamination migration on the Chandra X-ray Observatory - IV UV, X-Ray, and Gamma-Ray Space Instrumentation for Astronomy XX

SPIE Conference 10397 2017 August 6-8, San Diego, CA USA 


\section{Contaminant mass column Cool bake-out}

$$
\mathrm{T}_{\mathrm{FP}}=-60^{\circ} \mathrm{C} \quad \mathrm{T}_{\mathrm{DH}}=+25^{\circ} \mathrm{C} \quad \mathrm{T}_{\mathrm{OBF}}=+10^{\circ} \mathrm{C} \quad \mathrm{T}_{\pi \mathrm{T}}=-10^{\circ} \mathrm{C}
$$

$>$ Octadecane

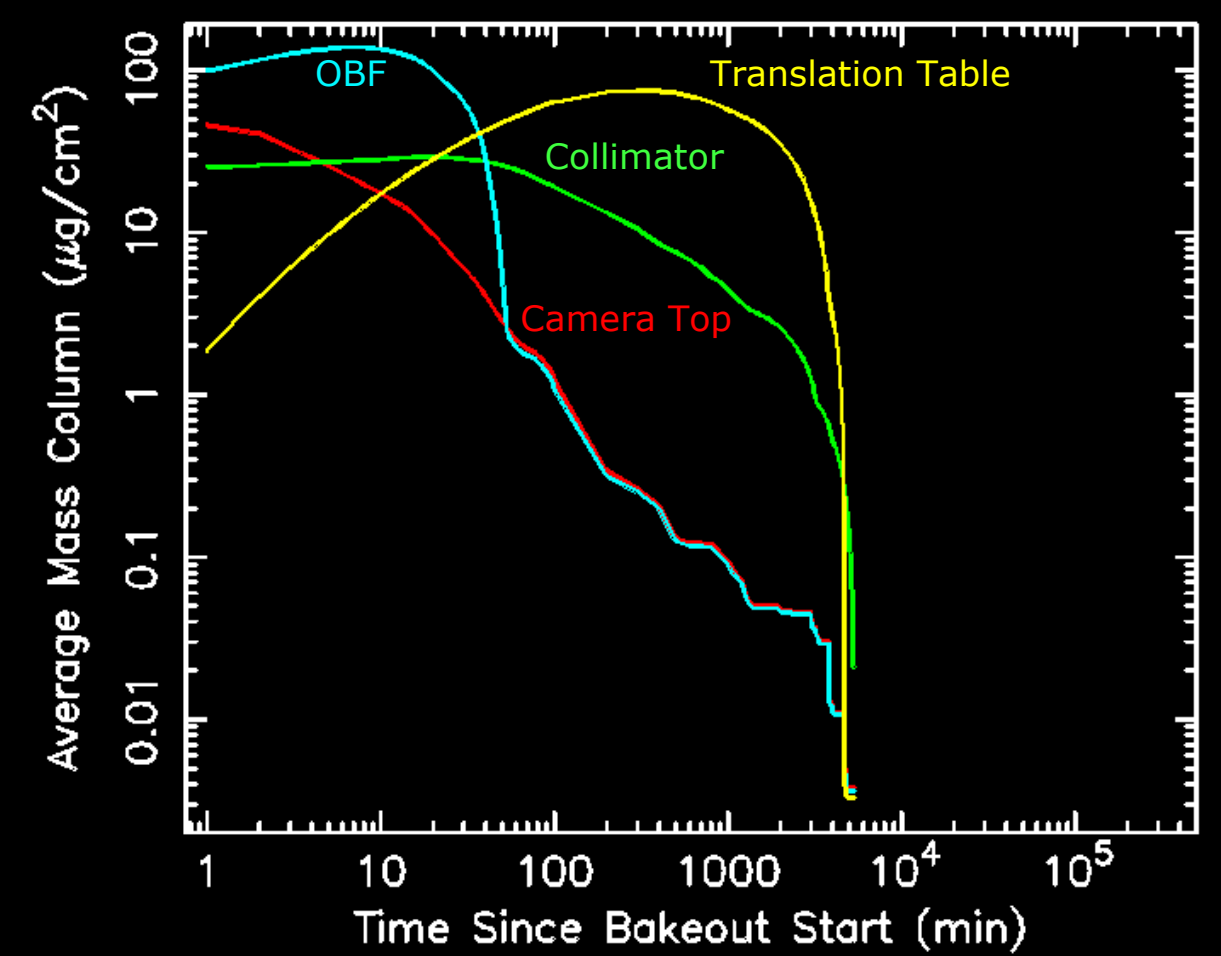

$>$ Dioctyl phthalate (DOP)

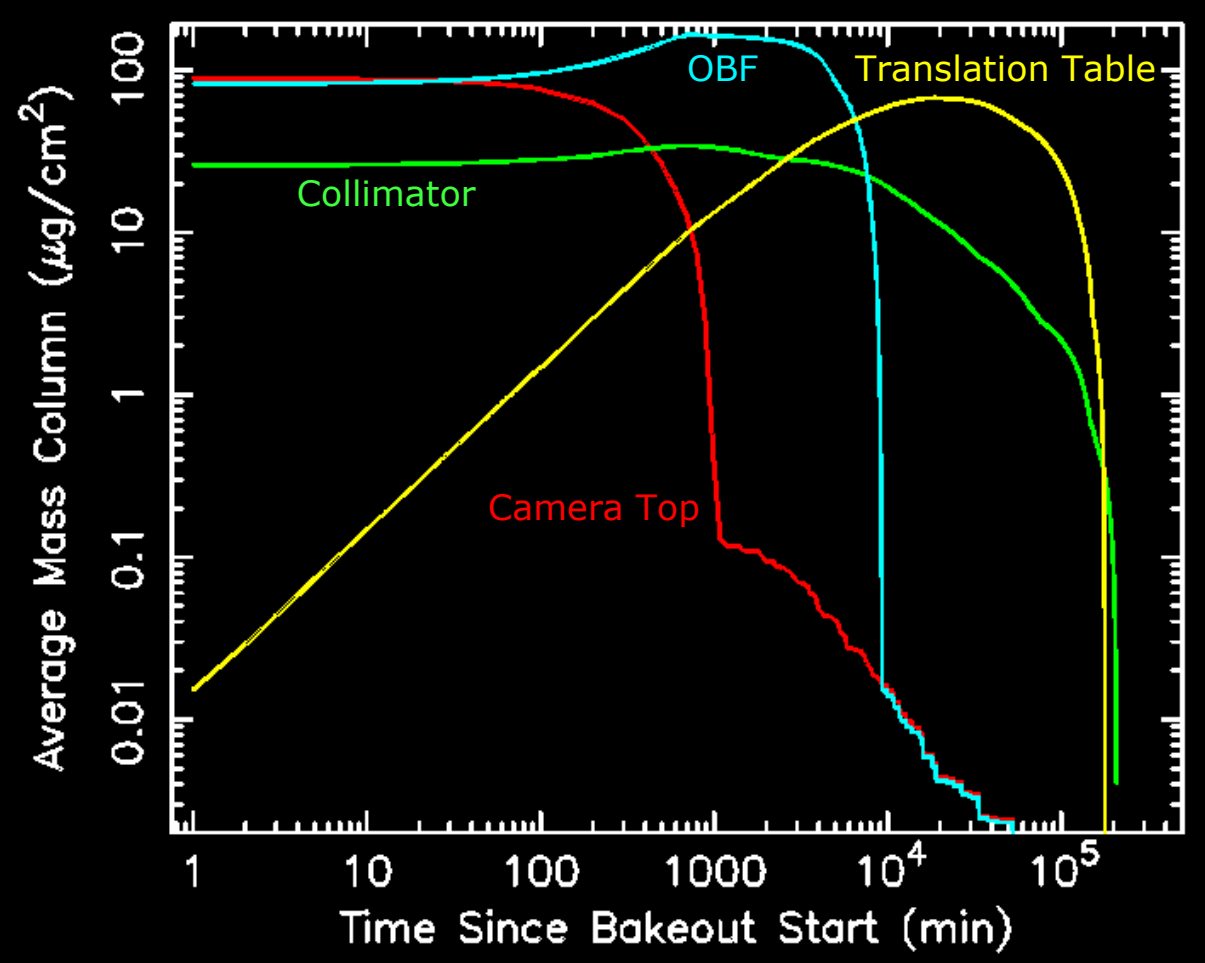

Modeling contamination migration on the Chandra X-ray Observatory - IV UV, X-Ray, and Gamma-Ray Space Instrumentation for Astronomy XX

SPIE Conference 10397 2017 August 6-8, San Diego, CA USA 


\section{Contaminant re-deposition after (partial) cool bake-out}

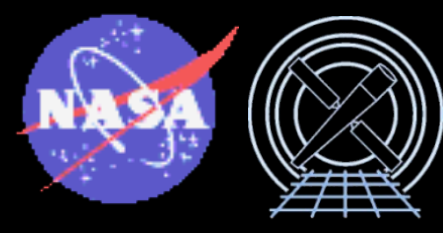

\section{Octadecane/10}

\section{Mass}

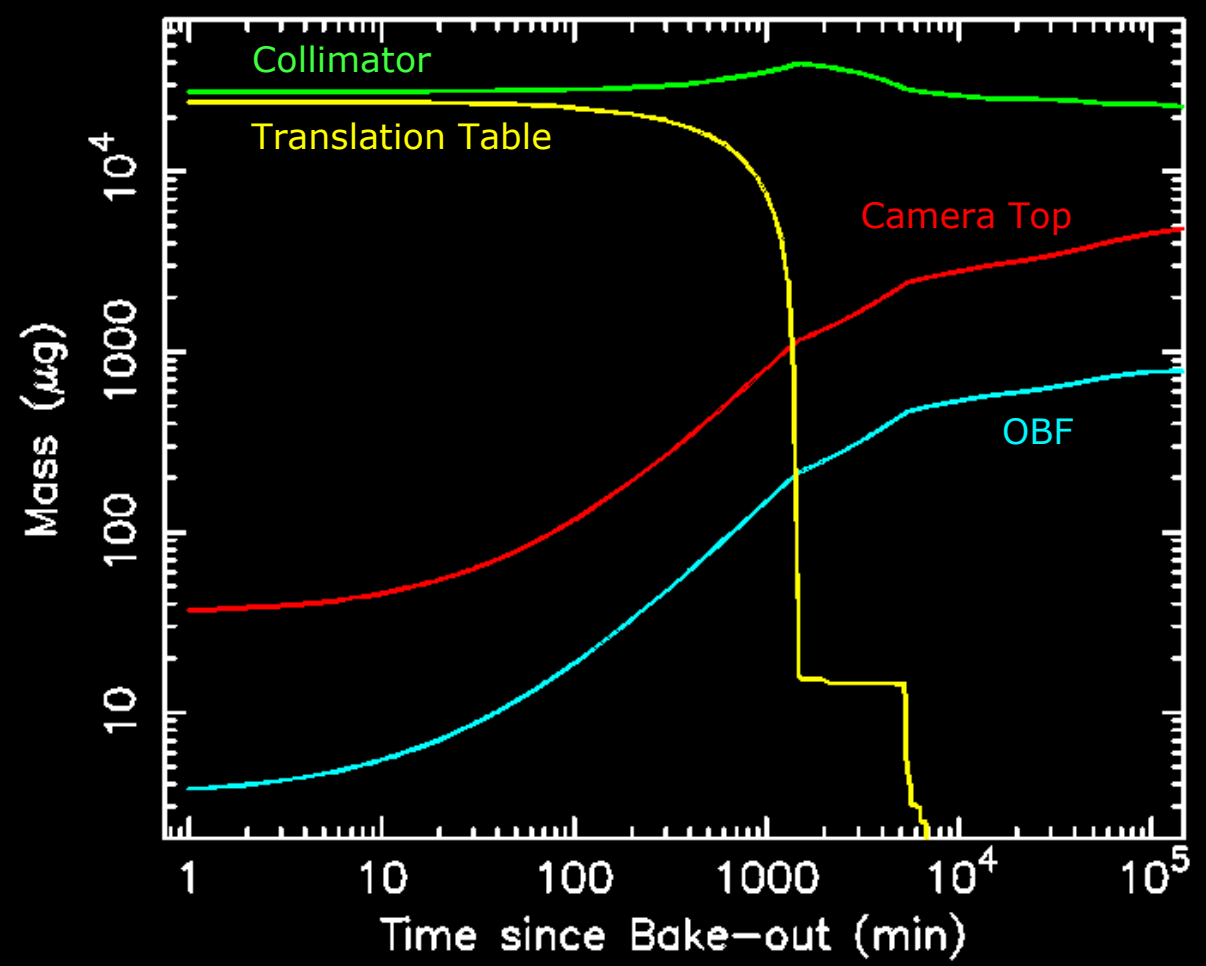

$>$ Mass column

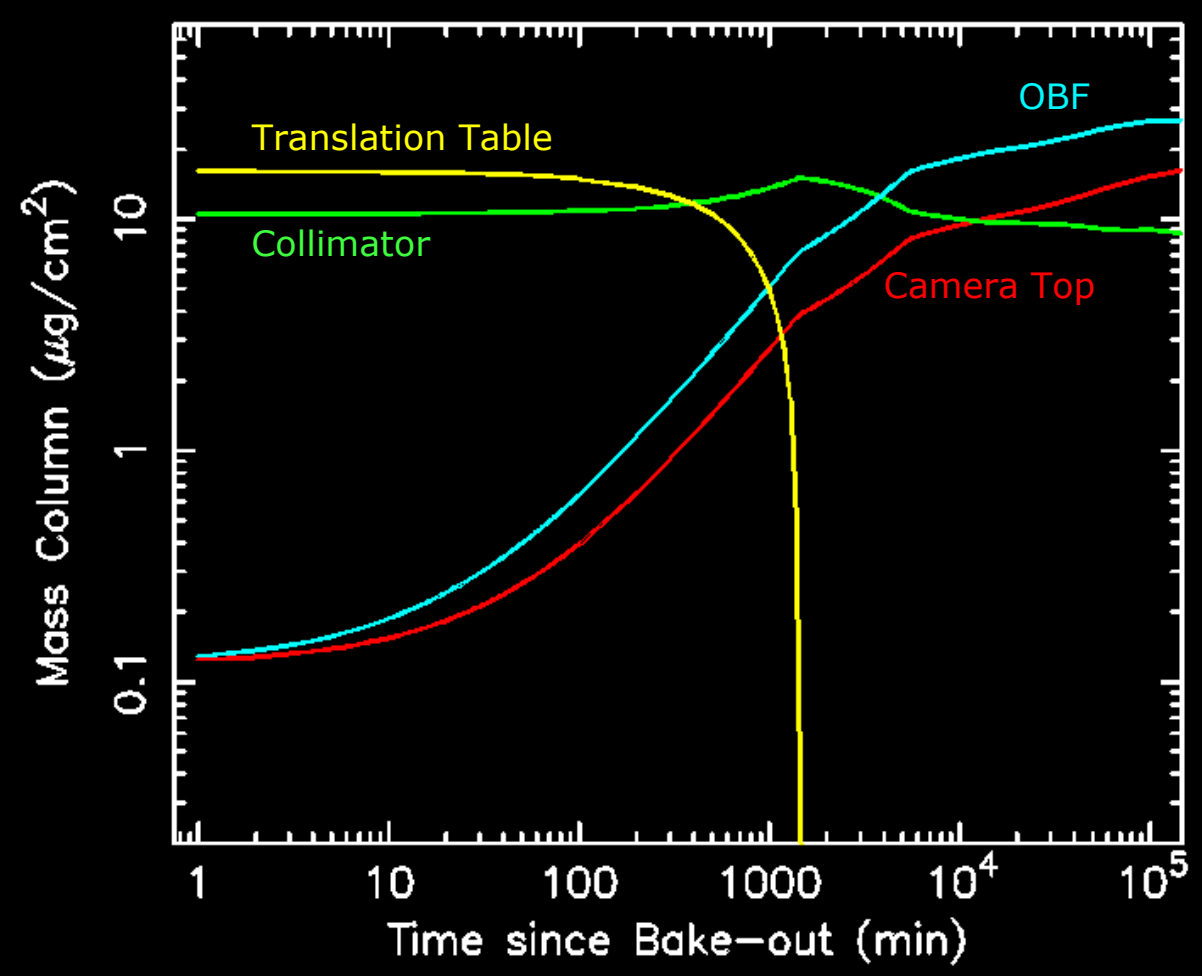

Modeling contamination migration on the Chandra X-ray Observatory - IV UV, X-Ray, and Gamma-Ray Space Instrumentation for Astronomy XX 2017 August 6-8, San Diego, CA USA
SPIE Conference 10397

Paper 13 Slide 16 


\section{Outline}

$>$ Introduction

$>$ Molecular contamination on ACIS filters

$>$ Thermal model for ACIS cavity

$>$ Molecular transport simulations

$>$ Summary 


\section{Summary}

$>$ Contamination-migration simulation provides a useful tool

口Utility for absolute predictions is still limited

- Absolute predictions require knowledge of contaminant's volatility

- Uncertainty in temperatures propagates exponentially to rate error

$\square$ Model may require additional physics

- Treatment of multiple molecular species is not simple superposition

- Dependence of thermal emissivity upon contaminant mass column

- Surface redistribution, especially for a liquid contaminant

$>$ Chandra Team has again deferred a decision to bake-out

$\square$ Scientific productivity continues despite low-energy absorption

- Observing proposals remain oversubscribed by factor $\approx 5.5$

- Over 400 refereed papers per year, steady over past decade

$\square$ Identified risks of performing bake-out are small but not zero

$\square$ Bake-out might not substantially reduce contamination on OBFs 